romanticism but not religion despite this Ontario-based poet's 1932/33 journal being suffused with religious imagery.

But these are small details, and small details significantly introduces us to fresh voices via meticulously edited entries. And, by offering us introductions to and diary entries by twenty women, small details makes available previously untold lives - and that is no small matter.

Connie Brim

University College of the Cariboo

Shenwen Li. Stratégies missionnaires des jésuites français en Nouvelle-France et en Chine au XVII ${ }^{e}$ siècle. Paris et Québec: L'Harmattan et Les Presses de l’Université Laval, 2001. Coll. InterCultures, 380 p.

Dans des articles parus en 1996 et 1998, Dominique Deslandres avait amorcé un travail comparatif au sujet des missions de la Nouvelle-France. D'une part, elle avait mis en parallèle les stratégies missionnaires déployées dans les missions intérieures de la France et celles utilisées, à la même époque, dans les missions amérindiennes; d'autre part, elle avait comparé les stratégies missionnaires mises en œuvre auprès des Amérindiens, en Amérique du Nord, par les Anglais et les Français. Dans le présent ouvrage, Shenwen Li ajoute une pierre à ce chantier comparatif dans le domaine des missions au XVII ${ }^{\mathrm{e}}$ siècle. Cette fois-ci, il tente d'établir une comparaison entre deux univers fort contrastés, le monde amérindien et la civilisation chinoise, deux univers où des jésuites français ont œuvré au $\mathrm{XVII}^{\mathrm{e}}$ siècle, bien que les périodes correspondant au sommet de leur activité dans ces deux mondes ne coïncident pas exactement.

Pour conduire ce travail comparatif, l'A. construit son ouvrage de manière assez classique. Une première partie (un chapitre) s'intéresse aux missionnaires jésuites : les origines, la structure hiérarchique et les règles de la compagnie, la formation des membres, l'implantation de la compagnie en France et la préparation aux deux missions d'outre-mer qui nous intéressent ici. Peut-être n'a-t-on pas porté ici une attention assez grande au 
contexte plus large : le catholicisme post-tridentin et l'évolution rapide de la réflexion missiologique dans l'Église catholique au moment où on institue la Propaganda fidei. Cela n'est pas neutre, surtout pour un ordre tel celui des jésuites tout entier dévoué aux volontés pontificales et de sa cour. Cela n'est pas sans intérêt non plus. En effet, l'évolution rapide de la pensée missiologique à cette époque peut conditionner les différences de stratégies observées, puisque les missions en Amérique du Nord et en Chine connaissent leur âge d'or à des périodes différentes : la première partie du XVII ${ }^{e}$ siècle en ce qui a trait à la Nouvelle-France (spécialement de 1632 à 1650) et au cours de la deuxième moitié de ce siècle, en Chine (spécialement de 1687 à 1717). Ce décalage, au moment où la pensée missiologique est en pleine évolution, n’est probablement pas anodin.

La deuxième et la troisième partie construisent deux tableaux symétriques. Le premier volet de ce diptyque (trois chapitres) est consacré aux missions jésuites en Nouvelle-France alors que le deuxième, construit de manière exactement parallèle, présente les missions jésuites dans l'Empire du Milieu (trois chapitres également). Si l'on décrit de manière satisfaisante les sociétés dans lesquelles se déploieront l'œuvre évangélisatrice (la société amérindienne au chapitre II et la société chinoise au chapitre V), on est sans doute insuffisamment sensible à d'autres dimensions qui ne sont pas neutres au regard des stratégies missionnaires adoptées : les cadres politiques, le cadre géographique (climat, densité de population, distances, etc.), le cadre politique local et géopolitique (société coloniale et concurrence Anglais-Français en Amérique du Nord), le contexte ecclésial (les essais missionnaires antérieurs à l'arrivée des jésuites auprès des mêmes populations, la concurrence entre les jésuites portugais et français en Chine), etc. Car, ce qui détermine les stratégies, ce ne sont pas seulement les sociétés rencontrées par les missionnaires, mais aussi tous les autres déterminants dans lesquels s'inscrit leur mission. En somme, on n'a pas que des missionnaires en présence de sociétés à " évangéliser », mais des missionnaires inscrits dans des jeux et des enjeux politiques, dans des contextes ecclésiaux, des géographies, etc. De plus, il serait intéressant d'examiner les hésitations et les discussions quant aux stratégies, aux méthodes et aux projets missionnaires à adopter plutôt que de trop fixer les choses. En fait, même chez les jésuites français en Nouvelle-France, il y a des tensions entre différents projets missionnaires possibles et toutes ces stratégies sont ouvertes à des évolutions commandées par des évolutions de 
contexte. La réduction et le séminaire ne sont pas les seules stratégies en présence.

La dernière partie (quatre chapitres) s’intéresse aux réactions des Amérindiens et des Chinois : les résistances et les oppositions, les conversions «à demy ", les phénomènes syncrétiques (quoique ce concept doive être utilisé avec beaucoup de précautions) et les « vrays chrestiens ».

La force de cette recherche, conduite suivant l'approche ethnologique, est de faire appel à des sources chinoises peu mises en valeur dans les études occidentales sur les missions chinoises. Cette étude, richement documentée, incitera peut-être d'autres chercheurs à entreprendre des recherches comparatives sur les missions. Déjà, Paul-André Dubois a mis en relation plusieurs stratégies missionnaires en usage dans les missions amérindiennes du nord avec celles qui avaient été expérimentées en Amérique méridionale. La recherche est donc à poursuivre.

Gilles Routhier

Faculté de théologie et de sciences religieuses

Université Laval

\section{Angela Nairne Grigor. Arthur Lismer, Visionary Art Educator. McGill-Queen's University Press, 2002. Pp. 349.}

When Canadians hear the name Arthur Lismer, they immediately think of the painter and member of the original Group of Seven. Angela Grigor's new book, Arthur Lismer, Visionary Art Educator is certainly going to change that perception. Grigor makes the very convincing case that Arthur Lismer was not only a member of the Group of Seven, who gave Canadian landscape painting a national identity, but he was also a resolute proponent of art education. Moreover, in his time, he defined himself and was identified by his influence on art education in Canada and around the world more than by his influence on painting. Although he was one of the most prominent art educators in Canada, his contribution has been largely ignored. This highly readable and insightful book should change not only the marginalization of the 